\title{
Classifying microscopic images as acute lymphoblastic leukemia by Resnet ensemble model and Taguchi method
}

\author{
Yao-Mei Chen ${ }^{1,2}$, Fu-I Chou ${ }^{3}$, Wen-Hsien $\mathrm{Ho}^{4,5^{*}}$ and Jinn-Tsong Tsai ${ }^{4,6^{*}}$ (])
}

From International Conference on Biomedical Engineering Innovation 2019 Kaohsiung, Taiwan. 15-19 November 2019

\author{
${ }^{*}$ Correspondence: \\ whho@kmu.edu.tw; \\ jttsai@mail.nptu.edu.tw \\ ${ }^{4}$ Department of Healthcare \\ Administration and Medical \\ Informatics, Kaohsiung \\ Medical University, \\ Kaohsiung 807, Taiwan \\ ${ }^{6}$ Department of Computer \\ Science, National Pingtung \\ University, Pingtung 900, \\ Taiwan \\ Full list of author information \\ is available at the end of the \\ article
}

\begin{abstract}
Background: Researchers have attempted to apply deep learning methods of artificial intelligence for rapidly and accurately detecting acute lymphoblastic leukemia (ALL) in microscopic images.

Results: A Resnet101-9 ensemble model was developed for classifying ALL in microscopic images. The proposed Resnet101-9 ensemble model combined the use of the nine trained Resnet-101 models with a majority voting strategy. Each trained Resnet-101 model integrated the well-known pre-trained Resnet-101 model and its algorithm hyperparameters by using transfer learning method to classify ALL in microscopic images. The best combination of algorithm hyperparameters for the pre-trained Resnet-101 model was determined by Taguchi experimental method. The microscopic images used for training of the pre-trained Resnet-101 model and for performance tests of the trained Resnet-101 model were obtained from the C-NMC dataset. In experimental tests of performance, the Resnet101-9 ensemble model achieved an accuracy of $85.11 \%$ and an $\mathrm{F}_{1}$-score of 88.94 in classifying ALL in microscopic images. The accuracy of the Resnet101-9 ensemble model was superior to that of the nine trained Resnet-101 individual models. All other performance measures (i.e., precision, recall, and specificity) for the Resnet101-9 ensemble model exceeded those for the nine trained Resnet-101 individual models.
\end{abstract}

Conclusion: Compared to the nine trained Resnet-101 individual models, the Resnet101-9 ensemble model had superior accuracy in classifying ALL in microscopic images obtained from the C-NMC dataset.

Keywords: Acute lymphoblastic leukemia, Microscopic image, ensemble model, Resnet model, Algorithm hyperparameter, Taguchi experimental method author(s) and the source, provide a link to the Creative Commons licence, and indicate if changes were made. The images or other third party material in this article are included in the article's Creative Commons licence, unless indicated otherwise in a credit line to the material. If material is not included in the article's Creative Commons licence and your intended use is not permitted by statutory regulation or exceeds the permitted use, you will need to obtain permission directly from the copyright holder. To view a copy of this licence, visit http:// creativecommons.org/licenses/by/4.0/. The Creative Commons Public Domain Dedication waiver (http://creativecommons.org/publi cdomain/zero/1.0/) applies to the data made available in this article, unless otherwise stated in a credit line to the data. 


\section{Background}

Acute lymphoblastic leukemia (ALL) is a cancer of the lymphoid line of blood cells characterized by development of numerous immature lymphocytes. As in acute leukemia, ALL progresses rapidly and is typically fatal within weeks or months if left untreated. If ALL is diagnosed in an early stage, however, curative treatment may be possible. Diagnosis is typically based on a complete blood count and microscope analysis of cell morphology, both of which are often performed manually by medical laboratory scientists. Although these tasks can be automated, the required equipment currently has a high cost and limited availability [1,2]. An automated system that uses relatively low-cost and easily obtained microscopic images for diagnosis of leukemia would have many advantages. Therefore, artificial intelligence models for automatically detecting ALL in microscopic images are urgently needed.

\section{Literature review}

Vogado et al. [3] extracted features from blood smear images by using pre-trained convolutional neural networks (CNNs) to obtain unique image descriptions. The authors evaluated several feature selection techniques and performed principal component analysis to select the features of the final descriptor. An ensemble model comprising support vector machine, multilayer perceptron, and random forest was then used to classify images as healthy or pathological. Rehman et al. [4] improved accuracy in diagnosing ALL by using a computer-aided system that integrated image processing and deep learning techniques. The authors proposed a method of classifying ALL in stained bone marrow images. Robust segmentation and deep learning techniques were used to train the CNN to classify bone marrow images accurately. Shafique and Tehsine [5] deployed a pre-trained AlexNet for automated detection and classification of ALL. The authors then classified ALL into subtypes L1, L2, and L3 in the French/American/British classification systems. The last layers of the pre-trained AlexNet were replaced with new layers for classifying input images into four classes: L1, L2, L3, and Normal. A data augmentation technique was also used to avoid overtraining. Liu and Long [1] proposed an ensemble model that used bagging ensemble learning method for training in ALL classification. The learning efficiency and classification accuracy of the proposed ensemble model was enhanced by using augmented images of ALL and elaborately designed training subsets for model training. In their preliminary test set, the proposed ensemble model obtained a weighted $\mathrm{F}_{1}$-score of 0.84 . Prellberg and Kramer [2] presented a simple and effective classification approach that used a ResNeXt CNN with squeeze-and-excitation modules. Preliminary tests of their approach in the C-NMC-2019 dataset achieved an average weighted $\mathrm{F}_{1}$-score of 0.8789 in 24 training runs. Kassani et al. [6] presented a hybrid system for automated classification of leukemic B-lymphoblasts. The hybrid system integrated two CNNs (VGG16 and MobileNet) and transfer learning to extract features from input images of leukemic B-lymphoblasts. The proposed system fused features from selected intermediate layers to obtain an auxiliary feature set, which was used for further improvement of classification accuracy. Additionally, features extracted from lower levels were integrated in higher dimension feature maps, which not only improved the capability to discriminate intermediate features, but also avoided the problem of 
vanishing/exploding network gradients. Loey et al. [7] proposed two automated classification models for detecting leukemia in blood microscopic images. Use of transfer learning in the two models yielded several advantages over traditional approaches. Their first classification model pre-processed blood microscopic images then used AlexNet, a pretrained deep CNN, for feature extraction. The AlexNet enabled application of numerous well-known classifiers. Their second classification model used pre-processed images to fine tune the AlexNet for both feature extraction and classification.

Notably, the above literature on detection of leukemia in microscopic images reveal that most related studies performed so far have investigated individual models for classifying microscopic images of ALL. Using an ensemble model, in which classification is based on the majority results, can reduce image classification errors. Moreover, few studies have discussed how algorithm hyperparameters affect classification accuracy in a pre-trained CNN model. Therefore, the motivations for this study were the lack of research on an ensemble model and lack of research on effect of algorithm hyperparameters on accuracy of a pre-trained $\mathrm{CNN}$ model.

\section{Objectives}

This study had two objectives. The first objective was to determine the best combination of algorithm hyperparameters for the pre-trained Resnet-101 model. The second objective of this study was to establish an ensemble model that used multiple trained Resnet-101 models and a majority voting strategy to classify ALL in microscopic images. The method of integrating an ensemble model and a majority voting strategy can solve the problem that different single models classify the same image with different symptoms. That is, classification of images by the ensemble model is analogous to classification of images according to the consensus of medical laboratory scientists. In a pre-trained Resnet-101 model, learning speed and classification quality are determined by algorithm hyperparameters that are set before the learning process begins. In subsequent training, however, a pre-trained Resnet-101 model may require different algorithm hyperparameters (e.g., optimizer, learning rate, and mini-batch size) to improve its classification accuracy. This study used Taguchi method, which is a systematic and robust experimental method, to generate the best combination of algorithm hyperparameters for a pre-trained Resnet-101 model. In experimental comparisons, the Resnet101 ensemble model had superior classification accuracy compared to trained Resnet-101 individual models and had excellent accuracy in classifying ALL in microscopic images.

\section{Problem description}

Acute lymphoblastic leukemia, a cancer type that affects the blood and bone marrow, is characterized by overproduction of immature white blood cells, called lymphoblasts or leukemic blasts. Since the bone marrow cannot produce adequate numbers of red cells, normal white cells, and platelets, people with ALL are susceptible to anemia and recurrent infections as well as easy bruising and bleeding. As a result, blast cells that spill out of the bone marrow and into the bloodstream can accumulate in various organs, including the lymph nodes (glands), spleen, liver, and central nervous system (brain and spinal cord) $[1,2]$. 


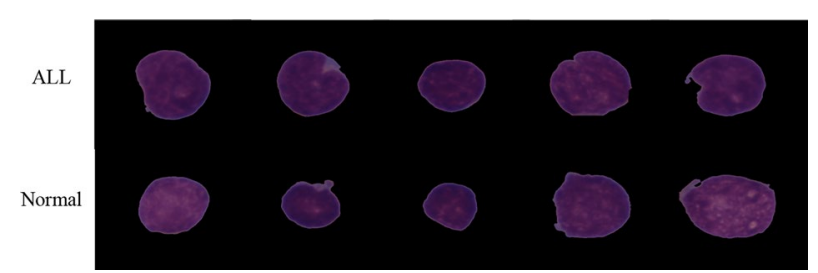

Fig. 1 Representative microscopic images of ALL cells and Normal cells

Table 1 Number of images in datasets for training and preliminary testing of performance in classifying ALL in microscopic images

\begin{tabular}{lccc}
\hline Class & Training set & Preliminary test set & Total images \\
\hline ALL & 7272 & 1219 & 8491 \\
Normal & 3389 & 648 & 4037 \\
Total images & 10,661 & 1867 & 12,528 \\
\hline
\end{tabular}

Acute lymphoblastic leukemia occurs in approximately $25 \%$ of all pediatric cancers. When viewed under a microscope, immature leukemic blasts and normal cells are difficult to distinguish due to their similar morphology [8]. Figure 1 compares representative microscopic images of ALL cells and Normal cells.

Detecting ALL often requires review of numerous and highly similar microscopic images by a medical laboratory scientist, which can be extremely time consuming and burdensome. Additionally, medical personnel in rural and/or under-developed areas may lack adequate training in detecting ALL in blood microscopic images. Therefore, the considered problem was how to screen numerous highly similar blood microscopic images for ALL efficiently and accurately. To aid medical laboratory personnel in identifying ALL in blood microscopic images, an artificial intelligence model trained by deep learning method may be a useful tool.

\section{Results}

The proposed Resnet101 ensemble model integrated multiple trained Resnet-101 models with a majority voting strategy for classifying ALL in microscopic images. The wellknown pre-trained Resnet-101 model with its appropriate algorithm hyperparameters was trained to classify ALL in microscopic images. The training set of microscopic images used to train the pre-trained Resnet-101 model was obtained from the C-NMC dataset. The preliminary test set of microscopic images was used for performance evaluation of the trained Resnet-101 model. The experimental environment was Matlab R2019 and its toolboxes developed by MathWorks.

The experimental data used to test performance in classifying ALL in microscopic images included the training set and the preliminary test set. Table 1 shows the number of microscopic images in the training set and in the preliminary test set. To maintain compatibility with the CNN-based architecture and the developed software, each microscopic image was processed as a $224 \times 224 \times 3$ image, where 3 is the number of color channels. 
Table 2 Three-level $L_{9}\left(3^{4}\right) \mathrm{OA}$

\begin{tabular}{lllll}
\hline Number of experiments & \multicolumn{2}{l}{ Factors } & C & D \\
\cline { 2 - 5 } & A & B & 1 & 1 \\
\hline 1 & 1 & 1 & 2 & 2 \\
2 & 1 & 2 & 3 & 3 \\
3 & 1 & 3 & 2 & 3 \\
4 & 2 & 1 & 3 & 1 \\
5 & 2 & 2 & 1 & 2 \\
6 & 2 & 3 & 3 & 2 \\
7 & 3 & 1 & 1 & 3 \\
8 & 3 & 2 & 2 & 1 \\
9 & 3 & 3 & & \\
\hline
\end{tabular}

Table 3 Factors and levels

\begin{tabular}{llll}
\hline Factor (Algorithm hyperparameter) & Levels & & \\
\cline { 2 - 4 } & $\mathbf{1}$ & $\mathbf{2}$ & $\mathbf{3}$ \\
\hline A: Optimizer & adam & sgdm & adam \\
B: MiniBatchSize & 60 & 65 & 70 \\
C: MaxEpochs & 8 & 10 & 12 \\
D: InitialLearnRate & $10^{-4}$ & $10^{-5}$ & $10^{-6}$ \\
\hline
\end{tabular}

A pre-trained Resnet-101 model was selected for training process, and then tried to set different algorithm hyperparameters before the learning process began. The algorithm hyperparameters for the pre-trained Resnet-101 model in this study were 'Optimizer', 'MiniBatchSize,' 'MaxEpochs', and 'InitialLearnRate.' Optimizer was the training option. MiniBatchSize was a mini-batch at each iteration. MaxEpochs was the maximum number of training epochs. InitialLearnRate was an option for decreasing the learning rate during training.

A three-level OA of the minimum number of experiments for four factors is $L_{9}\left(3^{4}\right)$. Table 2 shows the three-level $L_{9}\left(3^{4}\right)$ OA, and Table 3 shows the factors and levels. The three levels for the 'Optimizer' hyperparameter (factor A) were 'adam (adaptive moment estimation)', 'sgdm (stochastic gradient descent with a momentum)', and 'adam'. The three levels for the 'MiniBatchSize' hyperparameter (factor B) were 60, 65, and 70. The three levels for the 'MaxEpochs' hyperparameter (factor C) were 8, 10, and 12. The three levels for 'InitialLearnRate' hyperparameter (factor D) were $10^{-4}, 10^{-5}$, and $10^{-6}$. Instead of 81 $\left(3^{4}\right)$ experiments, the $L_{9}\left(3^{4}\right)$ OA required only 9 experiments.

Table 4 shows the combinations of the four algorithm hyperparameters that combined the values in Tables 2 and 3 and were used in a pre-trained Resnet-101 model for classifying ALL in microscopic images.

The algorithm hyperparameter combinations in Table 4 were used in three independent experimental runs in the training set of the pre-trained Resnet-101 model and in the preliminary test set of the trained Resnet-101 model. In tests of performance in classifying ALL in microscopic images, Table 5 shows the accuracy obtained in a single run and the average accuracy, standard deviation (SD), and $\eta$ value obtained in three runs. 
Table 4 Combinations of four algorithm hyperparameters for a pre-trained CNN model

\begin{tabular}{lllcl}
\hline $\begin{array}{l}\text { Number of } \\
\text { experiments }\end{array}$ & \multicolumn{4}{l}{ Algorithm hyperparameters } \\
\cline { 2 - 5 } & Optimizer & MiniBatchSize & MaxEpochs & InitialLearnRate \\
\hline 1 & adam & 60 & 8 & $10^{-4}$ \\
2 & adam & 65 & 10 & $10^{-5}$ \\
3 & adam & 70 & 12 & $10^{-6}$ \\
4 & sgdm & 60 & 10 & $10^{-6}$ \\
5 & sgdm & 65 & 12 & $10^{-4}$ \\
6 & sgdm & 70 & 8 & $10^{-5}$ \\
7 & adam & 60 & 12 & $10^{-5}$ \\
8 & adam & 65 & 8 & $10^{-6}$ \\
9 & adam & 70 & 10 & $10^{-4}$ \\
\hline
\end{tabular}

Table 5 Accuracy of the trained Resnet-101 model in classifying ALL in microscopic images when the algorithm hyperparameter combinations in Table 4 were used in three independent experimental runs

\begin{tabular}{|c|c|c|c|c|c|c|c|}
\hline \multirow{2}{*}{$\begin{array}{l}\text { Experiments } \\
1-9\end{array}$} & \multirow[t]{2}{*}{ Dataset } & \multicolumn{3}{|c|}{ Runs of experiment } & \multirow[t]{2}{*}{ Average accuracy } & \multirow[t]{2}{*}{ SD } & \multirow[t]{2}{*}{$\eta$ value } \\
\hline & & 1 & 2 & 3 & & & \\
\hline \multirow[t]{2}{*}{1} & Training set & 0.9777 & 0.9796 & 0.9792 & 0.9788 & 0.0010 & 33.4870 \\
\hline & Preliminary test set & 0.8045 & 0.8066 & 0.7927 & 0.8013 & 0.0075 & 14.0346 \\
\hline \multirow[t]{2}{*}{2} & Training set & 0.985 & 0.9864 & 0.9872 & 0.9862 & 0.0011 & 37.2024 \\
\hline & Preliminary test set & 0.7916 & 0.7943 & 0.805 & 0.7970 & 0.0071 & 13.8487 \\
\hline \multirow[t]{2}{*}{3} & Training set & 0.9211 & 0.9218 & 0.9216 & 0.9215 & 0.0004 & 22.1026 \\
\hline & Preliminary test set & 0.7477 & 0.7483 & 0.7483 & 0.7481 & 0.0003 & 11.9754 \\
\hline \multirow[t]{2}{*}{4} & Training set & 0.7892 & 0.7888 & 0.7893 & 0.7891 & 0.0003 & 13.5185 \\
\hline & Preliminary test set & 0.6508 & 0.6508 & 0.6508 & 0.6508 & 0.0000 & 9.1385 \\
\hline \multirow[t]{2}{*}{5} & Training set & 0.9533 & 0.9538 & 0.9535 & 0.9535 & 0.0003 & 26.6572 \\
\hline & Preliminary test set & 0.7783 & 0.7809 & 0.7788 & 0.7793 & 0.0014 & 13.1253 \\
\hline \multirow[t]{2}{*}{6} & Training set & 0.864 & 0.8639 & 0.8647 & 0.8642 & 0.0004 & 17.3420 \\
\hline & Preliminary test set & 0.6909 & 0.6904 & 0.6888 & 0.6900 & 0.0011 & 10.1737 \\
\hline \multirow[t]{2}{*}{7} & Training set & 0.985 & 0.9877 & 0.985 & 0.9859 & 0.0016 & 37.0156 \\
\hline & Preliminary test set & 0.8056 & 0.8013 & 0.7965 & 0.8011 & 0.0046 & 14.0288 \\
\hline \multirow[t]{2}{*}{8} & Training set & 0.9056 & 0.9057 & 0.9064 & 0.9059 & 0.0004 & 20.5282 \\
\hline & Preliminary test set & 0.7268 & 0.7327 & 0.7338 & 0.7311 & 0.0038 & 11.4082 \\
\hline \multirow[t]{2}{*}{9} & Training set & 0.9796 & 0.9831 & 0.9831 & 0.9819 & 0.0020 & 34.8624 \\
\hline & Preliminary test set & 0.7954 & 0.7868 & 0.7563 & 0.7795 & 0.0205 & 13.1318 \\
\hline
\end{tabular}

Table 6 Response table for each factor

\begin{tabular}{lllll}
\hline Level & \multicolumn{2}{l}{ Factors } & C & D \\
\cline { 2 - 5 } & A & B & 11.8722 & 13.4306 \\
\hline 1 & 13.2862 & 12.4006 & 12.0397 & 12.6837 \\
2 & 10.8125 & 12.7940 & 13.0432 & 10.8407 \\
3 & 12.8563 & 11.7603 & 1.1710 & 2.5898 \\
Effect & 2.4737 & 1.0337 & 13.0432 & 13.4306 \\
Maximum & 13.2862 & 12.7940 & 3 & 1 \\
Best level number & 1 & 2 & 12 & $10^{-4}$ \\
Best level value & adam & 65 & & \\
\hline
\end{tabular}




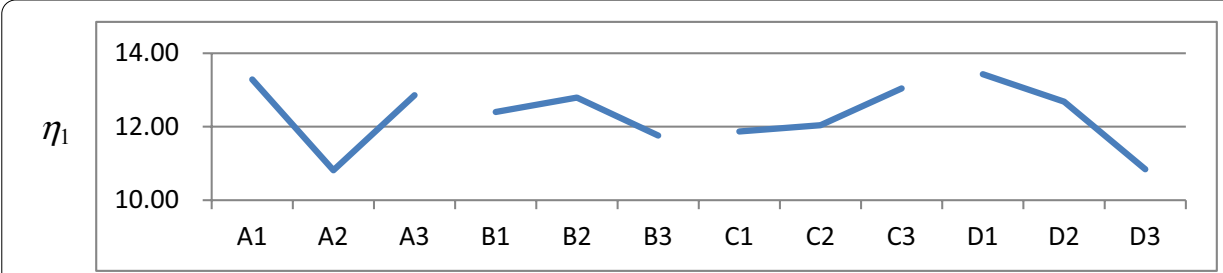

Level

Fig. 2 Plots of factor effects

Table 7 Accuracy of the nine trained Resnet-101 individual models in classifying ALL in microscopic images when the best combination of hyperparameters was used in nine independent experimental runs

\begin{tabular}{lcc}
\hline Model & Accuracy for the training set & $\begin{array}{c}\text { Accuracy for the } \\
\text { preliminary test } \\
\text { set }\end{array}$ \\
\hline Resnet-101-8249(\#1) & 0.9881 & 0.8249 \\
Resnet-101-8184(\#2) & 0.9856 & 0.8184 \\
Resnet-101-8452(\#3) & 0.9872 & 0.8452 \\
Resnet-101-8125(\#4) & 0.9893 & 0.8125 \\
Resnet-101-8061(\#5) & 0.9841 & 0.8061 \\
Resnet-101-8281(\#6) & 0.9848 & 0.8281 \\
Resnet-101-8307(\#7) & 0.9811 & 0.8307 \\
Resnet-101-8002(\#8) & 0.9877 & 0.8002 \\
Resnet-101-8216(\#9) & 0.9859 & 0.8216 \\
Average accuracy & 0.9860 & 0.8209 \\
SD & 0.0025 & 0.0136 \\
$\eta$ value & 37.0637 & 14.9359 \\
\hline
\end{tabular}

Table 6 is the response table for each factor, and Fig. 2 plots the effects of the factors, which were obtained by computing the $\eta$ value for each factor level in Table 5 . Table 6 shows that factor levels $1,2,3$, and 1 were selected for factors A, B, C, and D, respectively. Thus, the best factor-level combination was A1: adam, B2: 65, C3: 12, and D1: $10^{-4}$.

In the confirmation experiment, the best combination of algorithm hyperparameters (i.e., A1: adam, B2: 65, C3: 12, and D1: $10^{-4}$ ) was used in nine independent experimental runs of the trained Resnet-101 model, and the nine models were generated, namely Resnet-101-8249(\#1), Resnet-101-8184(\#2), Resnet-101-8452(\#3), Resnet-101-8125(\#4), Resnet-101-8061(\#5), Resnet-101-8281(\#6), Resnet-101-8307(\#7), Resnet-101-8002(\#8), and Resnet-101-8216(\#9). For classifying ALL in microscopic images, Table 7 shows the accuracy achieved in the nine models for the training set and the preliminary test set. Table 7 also shows that the average accuracy and $\eta$ value obtained in the nine models performed in the preliminary test set were 0.8209 and 14.9359 , respectively, which exceeded those in each $L_{9}\left(3^{4}\right)$ OA experiment (Table 5) in the preliminary test set. The best combination of algorithm hyperparameters in the response table can obtain the best result even though not all factor-level combinations were tested. Therefore, the best 
combination of algorithm hyperparameters obtained in the confirmation experiments was used in the trained Resnet-101 model for classifying ALL in microscopic images.

Additionally, ANOVA was performed to determine what factors had the greatest influence on the accuracy of the trained Resnet-101 model in classifying ALL in microscopic images. Table 8 shows the ANOVA results. Factors A (optimizer) and D (initialLearnRate) had the largest effects on accuracy. The percentage contributions of Factors A and D to experimental variance were $41.62 \%$ and $42.34 \%$, respectively, and their total percentage contribution to experimental variance was $83.96 \%$. Therefore, statistically significant factors in the accuracy of the trained Resnet-101 model in classifying ALL in microscopic images were A1 (adam) and D1 $\left(10^{-4}\right)$.

Table 7 shows the high classification accuracy achieved by each model in the preliminary test set. In all models, classification accuracy in the preliminary test set exceeded that of all $L_{9}\left(3^{4}\right)$ OA experiments (Table 5). Therefore, the nine trained Resnet-101 individual models were selected for inclusion in the ensemble model. The Resnet101 ensemble models comprised four ensemble models (Resnet101-3, Resnet101-5, Resnet101-7, and Resnet101-9). A microscopic image classified as ALL cell by most models was considered an ALL class, and a microscopic image classified as Normal cell by most models was considered a Normal class. The accuracy metric was used to compare performance in the trained Resnet-101 and Resnet101 ensemble models. Precision, recall, specificity, and $\mathrm{F}_{1}$-score were further used to validate classification performance. The results were depicted by creating a confusion matrix of actual class versus predicted class for the respective classes.

The comparison of classification accuracy in the four ensemble models in the preliminary test set were as follows.

The Resnet101-3 ensemble model, which combined Resnet-101-8249, Resnet-101-8184, and Resnet-101-8452 models, classified ALL in microscopic images by aggregating the results of a majority voting strategy. Table 9 shows the confusion matrices used to compare ALL and Normal classes in the Resnet-101-8249, Resnet-101-8184, Resnet-101-8452, and Resnet101-3 ensemble models for the preliminary test set.

Based on the data in Table 9, Table 10 shows the classifier accuracy, precision, recall, specificity, and $\mathrm{F}_{1}$-score obtained by the trained Resnet- 101 individual models and by the Resnet101-3 ensemble model. When the preliminary test set was used, the accuracy of the Resnet101-3 ensemble model (0.8409) was higher than those of the Resnet-101-8249 and Resnet-101-8184 models but lower than that of the Resnet-101-8452 model.

Table 8 Summary of ANOVA results

\begin{tabular}{lclccc}
\hline Factor & Sum of squares & $\begin{array}{l}\text { Degrees of } \\
\text { freedom }\end{array}$ & Variance & $\begin{array}{l}\text { Expected sum } \\
\text { of squares }\end{array}$ & $\begin{array}{l}\text { Percentage } \\
\text { contribution } \\
\text { (\%) }\end{array}$ \\
\hline A: optimizer & 10.4812 & 2 & 5.2406 & 10.4812 & 41.62 \\
B: miniBatchSize & 1.6333 & 2 & 0.8167 & 1.6333 & 6.49 \\
C: maxEpochs & 2.4063 & 2 & 1.2031 & 2.4063 & 9.56 \\
D: initialLearnRate & 10.6617 & 2 & 5.3309 & 10.6617 & 42.34 \\
Error & 0.0000 & 0 & & & 100 \\
$S_{T}$ & 25.1825 & 8 & & & \\
\hline
\end{tabular}


Table 9 Confusion matrix for classification of images as ALL or Normal classes by the trained Resnet-101 individual models and by the Resnet101-3 ensemble model for the preliminary test set

\begin{tabular}{|c|c|c|c|c|}
\hline \multirow[t]{2}{*}{ Model } & & & \multicolumn{2}{|c|}{ Actual classes } \\
\hline & & & $\overline{A L L}$ & Normal \\
\hline \multirow{2}{*}{ Resnet-101-8249 } & Predicted classes & ALL & 1095 & 203 \\
\hline & & Normal & 124 & 445 \\
\hline \multirow[t]{2}{*}{ Resnet-101-8184 } & Predicted classes & ALL & 1047 & 167 \\
\hline & & Normal & 172 & 481 \\
\hline \multirow[t]{2}{*}{ Resnet-101-8452 } & Predicted classes & ALL & 1114 & 184 \\
\hline & & Normal & 105 & 464 \\
\hline \multirow[t]{2}{*}{ Resnet101-3 ensemble } & Predicted classes & ALL & 1104 & 182 \\
\hline & & Normal & 115 & 466 \\
\hline
\end{tabular}

Table 10 Classification accuracy, precision, recall, specificity, and $F_{1}$-score obtained by trained Resnet-101 individual models and by the Resnet101-3 ensemble model for the preliminary test set

\begin{tabular}{llllll}
\hline Model & Accuracy & Precision & Recall & Specificity & $\mathbf{F}_{1}$-score \\
\hline Resnet-101-8249 & 0.8249 & 0.8436 & 0.8983 & 0.6867 & 0.8701 \\
Resnet-101-8184 & 0.8184 & 0.8624 & 0.8589 & 0.7423 & 0.8607 \\
Resnet-101-8452 & 0.8452 & 0.8582 & 0.9139 & 0.7160 & 0.8852 \\
Resnet101-3 ensemble & 0.8409 & 0.8585 & 0.9057 & 0.7191 & 0.8814 \\
\hline
\end{tabular}

Table 11 Confusion matrix for classification of images as ALL and Normal classes by the trained Resnet-101 individual models and by the Resnet101-5 ensemble model for the preliminary test set

\begin{tabular}{|c|c|c|c|c|}
\hline \multirow[t]{2}{*}{ Model } & & & \multicolumn{2}{|c|}{ Actual classes } \\
\hline & & & ALL & Normal \\
\hline \multirow[t]{2}{*}{ Resnet-101-8249 } & Predicted classes & ALL & 1095 & 203 \\
\hline & & Normal & 124 & 445 \\
\hline \multirow[t]{2}{*}{ Resnet-101-8184 } & Predicted classes & $A L L$ & 1047 & 167 \\
\hline & & Normal & 172 & 481 \\
\hline \multirow[t]{2}{*}{ Resnet-101-8452 } & Predicted classes & $A L L$ & 1114 & 184 \\
\hline & & Normal & 105 & 464 \\
\hline \multirow[t]{2}{*}{ Resnet-101-8125 } & Predicted classes & $A L L$ & 1078 & 209 \\
\hline & & Normal & 141 & 439 \\
\hline \multirow[t]{2}{*}{ Resnet-101-8061 } & Predicted classes & $A L L$ & 1030 & 173 \\
\hline & & Normal & 189 & 475 \\
\hline \multirow[t]{2}{*}{ Resnet101-5 ensemble } & Predicted classes & ALL & 1100 & 173 \\
\hline & & Normal & 119 & 475 \\
\hline
\end{tabular}

Other performance measures (i.e., precision, recall, specificity, and $F_{1}$-score) for the Resnet101-3 ensemble model approximated those in the trained Resnet-101 individual models.

The Resnet101-5 ensemble model applied a majority voting strategy to classify ALL in microscopic images. That is, the Resnet101-5 ensemble model classified ALL by aggregating the results of five models: Resnet-101-8249, Resnet-101-8184, Resnet-101-8452, 
Resnet-101-8125, and Resnet-101-8061 models. Table 11 shows the confusion matrices used for comparisons of ALL and Normal classes in the Resnet-101-8249, Resnet-101-8184, Resnet-101-8452, Resnet-101-8125, Resnet-101-8061, and Resnet101-5 ensemble models for the preliminary test set.

Based on the data in Table 11, Table 12 shows the classifier accuracy, precision, recall, specificity, and $\mathrm{F}_{1}$-score obtained by the trained Resnet-101 individual models and by the Resnet101-5 ensemble model. In the preliminary test set, the accuracy of the Resnet101-5 ensemble model (0.8436) was superior to that of all trained Resnet-101 individual models except Resnet-101-8452 model. Other performance measures (i.e., precision, recall, specificity, and $F_{1}$-score) obtained by the Resnet101-5 ensemble model were not consistently superior or inferior to those of the trained Resnet-101 individual models.

As in the Resnet101-7 ensemble model, the Resnet101-7 ensemble model used a majority voting strategy to classify ALL in microscopic images. However, the Resnet-7 ensemble model integrated seven models: Resnet-101-8249, Resnet-101-8184, Resnet-101-8452, Resnet-101-8125, Resnet-101-8061, Resnet-101-8281, and Resnet-101-8307 models. The Resnet101-7 ensemble model classified ALL by aggregating the results of the majority voting strategy. Table 13 shows the confusion matrices used to compare performance in classifying images in the preliminary test set as ALL or Normal classes in the seven trained Resnet-101 individual models and in the Resnet101-7 ensemble model.

Based on the data in Table 13, Table 14 shows the classification accuracy, precision, recall, specificity, and $\mathrm{F}_{1}$-score obtained by the trained Resnet-101 individual models and by the Resnet101-7 ensemble model. In the preliminary test set, the Resnet101-7 ensemble model achieved an accuracy of 0.8506 , which was superior to those of the trained Resnet-101 individual models. All other performance measures (i.e., precision, recall, specificity, and $\mathrm{F}_{1}$-score) obtained for the Resnet101-7 ensemble model were higher than those for the trained Resnet-101 individual models. That is, the Resnet101-7 ensemble model had superior accuracy in classifying ALL in microscopic images.

The Resnet101-9 ensemble model, which combined Resnet-101-8249, Resnet-101-8184, Resnet-101-8452, Resnet-101-8125, Resnet-101-8061, Resnet-101-8281, Resnet-101-8307, Resnet-101-8002, and Resnet-101-8216 models, accurately classified ALL in microscopic images by using a majority voting strategy to aggregate the results of these nine models. Table 15 shows the confusion matrices used

Table 12 Classification accuracy, precision, recall, specificity, and $F_{1}$-score obtained by trained Resnet-101 individual models and by the Resnet101-5 ensemble model for the preliminary test set

\begin{tabular}{|c|c|c|c|c|c|}
\hline Model & Accuracy & Precision & Recall & Specificity & $F_{1}$-score \\
\hline Resnet-101-8249 & 0.8249 & 0.8436 & 0.8983 & 0.6867 & 0.8701 \\
\hline Resnet-101-8184 & 0.8184 & 0.8624 & 0.8589 & 0.7423 & 0.8607 \\
\hline Resnet-101-8452 & 0.8452 & 0.8582 & 0.9139 & 0.716 & 0.8852 \\
\hline Resnet-101-8125 & 0.8125 & 0.8376 & 0.8843 & 0.6775 & 0.8603 \\
\hline Resnet-101-8061 & 0.8061 & 0.8562 & 0.845 & 0.733 & 0.8505 \\
\hline Resnet101-5 ensemble & 0.8436 & 0.8641 & 0.9024 & 0.733 & 0.8828 \\
\hline
\end{tabular}


Table 13 Confusion matrix for classification of images as ALL and Normal classes by the trained Resnet-101 individual models and by the Resnet101-7 ensemble model for the preliminary test set

\begin{tabular}{|c|c|c|c|c|}
\hline \multirow[t]{2}{*}{ Model } & & & \multicolumn{2}{|c|}{ True classes } \\
\hline & & & ALL & Normal \\
\hline \multirow[t]{2}{*}{ Resnet-101-8249 } & Predicted classes & ALL & 1095 & 203 \\
\hline & & Normal & 124 & 445 \\
\hline \multirow[t]{2}{*}{ Resnet-101-8184 } & Predicted classes & ALL & 1047 & 167 \\
\hline & & Normal & 172 & 481 \\
\hline \multirow[t]{2}{*}{ Resnet-101-8452 } & Predicted classes & ALL & 1114 & 184 \\
\hline & & Normal & 105 & 464 \\
\hline \multirow[t]{2}{*}{ Resnet-101-8125 } & Predicted classes & ALL & 1078 & 209 \\
\hline & & Normal & 141 & 439 \\
\hline \multirow[t]{2}{*}{ Resnet-101-8061 } & Predicted classes & ALL & 1030 & 173 \\
\hline & & Normal & 189 & 475 \\
\hline \multirow[t]{2}{*}{ Resnet-101-8281 } & Predicted classes & ALL & 1114 & 216 \\
\hline & & Normal & 105 & 432 \\
\hline \multirow[t]{2}{*}{ Resnet-101-8307 } & Predicted classes & ALL & 1090 & 187 \\
\hline & & Normal & 129 & 461 \\
\hline \multirow[t]{2}{*}{ Resnet101-7 ensemble } & Predicted classes & ALL & 1116 & 176 \\
\hline & & Normal & 103 & 472 \\
\hline
\end{tabular}

Table 14 Classification accuracy, precision, recall, specificity, and $F_{1}$-score obtained by trained Resnet-101 individual models and by the Resnet101-7 ensemble model for the preliminary test set

\begin{tabular}{llllll}
\hline Model & Accuracy & Precision & Recall & Specificity & F $_{1}$-score \\
\hline Resnet-101-8249 & 0.8249 & 0.8436 & 0.8983 & 0.6867 & 0.8701 \\
Resnet-101-8184 & 0.8184 & 0.8624 & 0.8589 & 0.7423 & 0.8607 \\
Resnet-101-8452 & 0.8452 & 0.8582 & 0.9139 & 0.716 & 0.8852 \\
Resnet-101-8125 & 0.8125 & 0.8376 & 0.8843 & 0.6775 & 0.8603 \\
Resnet-101-8061 & 0.8061 & 0.8562 & 0.845 & 0.733 & 0.8505 \\
Resnet-101-8281 & 0.8281 & 0.8376 & 0.9139 & 0.6667 & 0.8741 \\
Resnet-101-8307 & 0.8307 & 0.8536 & 0.8942 & 0.7114 & 0.8734 \\
Resnet101-7 ensemble & 0.8506 & 0.8638 & 0.9155 & 0.7284 & 0.8889 \\
\hline
\end{tabular}

for comparisons of ALL and Normal classes. Table 15 is the confusion matrix for the classification performance of the nine trained Resnet-101 individual models and the Resnet101-9 ensemble model for the preliminary test set.

Based on the data in Table 15, Table 16 shows the classifier accuracy, precision, recall, specificity, and $\mathrm{F}_{1}$-score obtained by the trained Resnet-101 individual models and by the Resnet101-9 ensemble model. When the Resnet101-9 ensemble model was used in the preliminary test set, the accuracy was 0.8511 , which was superior to the accuracies obtained by the trained Resnet-101 individual models. Other performance measures (i.e., precision, recall, specificity, and $F_{1}$-score) obtained by the Resnet101-9 ensemble model were higher than those obtained by the trained Resnet-101 individual models. 
Table 15 Confusion matrix for performance of the trained Resnet-101 individual models and the Resnet101-9 ensemble model in classifying images in the preliminary test set as ALL or Normal classes

\begin{tabular}{|c|c|c|c|c|}
\hline \multirow[t]{2}{*}{ Model } & & & \multicolumn{2}{|c|}{ Actual classes } \\
\hline & & & $\overline{A L L}$ & Normal \\
\hline \multirow[t]{2}{*}{ Resnet-101-8249 } & Predicted classes & ALL & 1095 & 203 \\
\hline & & Normal & 124 & 445 \\
\hline \multirow[t]{2}{*}{ Resnet-101-8184 } & Predicted classes & ALL & 1047 & 167 \\
\hline & & Normal & 172 & 481 \\
\hline \multirow[t]{2}{*}{ Resnet-101-8452 } & Predicted classes & ALL & 1114 & 184 \\
\hline & & Normal & 105 & 464 \\
\hline \multirow[t]{2}{*}{ Resnet-101-8125 } & Predicted classes & ALL & 1078 & 209 \\
\hline & & Normal & 141 & 439 \\
\hline \multirow[t]{2}{*}{ Resnet-101-8061 } & Predicted classes & ALL & 1030 & 173 \\
\hline & & Normal & 189 & 475 \\
\hline \multirow[t]{2}{*}{ Resnet-101-8281 } & Predicted classes & ALL & 1114 & 216 \\
\hline & & Normal & 105 & 432 \\
\hline \multirow[t]{2}{*}{ Resnet-101-8307 } & Predicted classes & ALL & 1090 & 187 \\
\hline & & Normal & 129 & 461 \\
\hline \multirow[t]{2}{*}{ Resnet-101-8002 } & Predicted classes & ALL & 1032 & 186 \\
\hline & & Normal & 187 & 462 \\
\hline \multirow[t]{2}{*}{ Resnet-101-8216 } & Predicted classes & ALL & 1099 & 213 \\
\hline & & Normal & 120 & 435 \\
\hline \multirow[t]{2}{*}{ Resnet101-9 ensemble } & Predicted classes & ALL & 1118 & 177 \\
\hline & & Normal & 101 & 471 \\
\hline
\end{tabular}

Table 16 Classification accuracy, precision, recall, specificity, and $\mathrm{F}_{1}$-score obtained by the trained Resnet-101 individual models and by the Resnet101-9 ensemble model for the preliminary test set

\begin{tabular}{llllll}
\hline Model & Accuracy & Precision & Recall & Specificity & F $_{1}$-score \\
\hline Resnet-101-8249 & 0.8249 & 0.8436 & 0.8983 & 0.6867 & 0.8701 \\
Resnet-101-8184 & 0.8184 & 0.8624 & 0.8589 & 0.7423 & 0.8607 \\
Resnet-101-8452 & 0.8452 & 0.8582 & 0.9139 & 0.716 & 0.8852 \\
Resnet-101-8125 & 0.8125 & 0.8376 & 0.8843 & 0.6775 & 0.8603 \\
Resnet-101-8061 & 0.8061 & 0.8562 & 0.845 & 0.733 & 0.8505 \\
Resnet-101-8281 & 0.8281 & 0.8376 & 0.9139 & 0.6667 & 0.8741 \\
Resnet-101-8307 & 0.8307 & 0.8536 & 0.8942 & 0.7114 & 0.8734 \\
Resnet-101-8002 & 0.8002 & 0.8473 & 0.8466 & 0.7130 & 0.8469 \\
Resnet-101-8216 & 0.8216 & 0.8377 & 0.9016 & 0.6713 & 0.8684 \\
Resnet101-9 ensemble & 0.8511 & 0.8633 & 0.9171 & 0.7269 & 0.8894 \\
\hline
\end{tabular}

That is, the Resnet101-9 ensemble model had superior accuracy in classifying ALL in microscopic images.

The classification results from Resent101-3, -5, -7, -9 ensemble models showed that the classification accuracy of multiple integrated models was higher than that of a single model and fewer integrated models. In this study, the ensemble models that integrated the largest number of models (i.e., nine models) had the highest classification 
Table 17 Image classification errors by the Resnet101-9 ensemble model for the preliminary test set

\begin{tabular}{|c|c|c|c|}
\hline Classification error status & $\begin{array}{l}\text { Number of } \\
\text { incorrect } \\
\text { classifications }\end{array}$ & Numbers of microscopic images & $\begin{array}{l}\text { Amount of } \\
\text { incorrect } \\
\text { classifications }\end{array}$ \\
\hline & 5 & $\begin{array}{l}165,261,279,355,368,388,533,570 \\
574,632,690,857,1010,1095,1235 \\
1254,1301,1355,1522,1606,1625 \\
1682,1709,1715\end{array}$ & 24 \\
\hline & 6 & $\begin{array}{l}294,377,528,544,908,912,1099,1219 \\
1408,1433\end{array}$ & 10 \\
\hline ALL incorrectly classified as & 7 & $\begin{array}{l}210,447,525,629,646,767,799,805 \\
855,882,887,913,1132,1223,1405 \\
1861\end{array}$ & 16 \\
\hline \multirow[t]{4}{*}{ Normal } & 8 & $\begin{array}{l}250,389,433,612,746,976,1031,1127 \\
1277,1361,1492,1515,1521,1652 \\
1692\end{array}$ & 15 \\
\hline & 9 & $\begin{array}{l}47,179,204,219,239,295,336,427 \\
634,692,719,737,768,843,850,859 \\
869,910,961,1019,1081,1116,1121 \\
1310,1337,1397,1418,1434,1528 \\
1531,1580,1588,1592,1769,1796 \\
1834\end{array}$ & 36 \\
\hline & 5 & $\begin{array}{l}60,63,67,90,127,187,240,391,431 \\
461,465,567,787,891,946,1335,1365 \\
1367,1441,1449,1485,1487,1514 \\
1538,1634,1723,1739,1758,1823 \\
1865\end{array}$ & 30 \\
\hline & 6 & $\begin{array}{l}158,173,233,258,305,313,376,405 \\
442,464,728,747,814,866,872,933 \\
1062,1074,1123,1149,1275,1591 \\
1603,1629,1696,1729,1787\end{array}$ & 27 \\
\hline Normal incorrectly classified as & 7 & $\begin{array}{l}236,251,298,382,446,475,516,693 \\
698,724,898,1111,1126,1175,1195 \\
1265,1295,1377,1399,1431,1473 \\
1530,1716,1815\end{array}$ & 24 \\
\hline \multirow[t]{2}{*}{ ALL } & 8 & $\begin{array}{l}13,172,220,289,420,435,484,529 \\
627,684,775,831,949,1063,1119 \\
1247,1263,1379,1411,1537,1545 \\
1590,1624,1673,1732,1759,1820 \\
1840,1850\end{array}$ & 29 \\
\hline & 9 & $\begin{array}{l}26,35,50,54,117,142,160,171,212 \\
214,256,259,264,299,320,340,369 \\
421,423,469,472,530,531,536,609 \\
643,654,682,735,786,791,840,854 \\
864,867,896,924,930,931,963,974 \\
980,996,1017,1072,1191,1220,1222 \\
1249,1252,1267,1307,1324,1409 \\
1422,1440,1458,1460,1525,1526 \\
1623,1724,1741,1749,1773,1786 \\
1814\end{array}$ & 67 \\
\hline
\end{tabular}

Number of incorrect classifications: The number of incorrect classifications of an image by the nine individual models

accuracy. Therefore, the Resnet101-9 ensemble model combined with a majority voting strategy was used to classify ALL in microscopic images.

\section{Discussion}

The preliminary test set contained 1867 microscopic images, including 1219 images of ALL cells and 648 images of Normal cells. Table 17 shows the numbers of images that the Resnet101-9 ensemble model classified incorrectly. 101 microscopic images were 
ALL cells but were incorrectly classified as Normal cells, and 36 images were incorrectly classified by nine individual models and should be reviewed by medical laboratory scientists. 177 microscopic images were Normal cells but were incorrectly classified as ALL cells, and 67 images were incorrectly classified by nine individual models and should be reviewed by medical laboratory scientists. Microscopic images of acute lymphoblastic leukemia were obtained from the C-NMC dataset for testing the performance of the proposed artificial intelligence methods. Classification accuracy of the preliminary test set in the previous studies $[1,2,9]$ and the Resnet101-9 ensemble model is no more than 0.9. The authors coming from Medical University believed that some images from the C-NMC dataset were incorrectly labeled and needed further confirmation by medical laboratory scientists. In most object detection and classification problems encountered in the medical field, professional knowledge or experience is needed to label objects correctly. Therefore, a reliable dataset of correctly labeled objects is essential for model training and testing.

This study found that an appropriate combination of algorithm hyperparameter settings for a pre-trained Resnet-101 model is essential for accurately classifying ALL in microscopic images. In the trained Resnet-101 model, the best combination was Optimizer of 'adam', MiniBatchSize of 65, MaxEpochs of 12, and InitialLearnRate of $10^{-4}$. The results of this study indicate that a poor combination of algorithm hyperparameters for a pre-trained Resnet-101 model cannot accurately classify ALL in microscopic images. Although different trained Resnet-101 individual models have different accuracy in classifying ALL in microscopic images, the Resnet101 ensemble model used a voting mechanism to aggregate the classification results. That is, classification of microscopic images of ALL by the ensemble model is analogous to classification of microscopic images of ALL according to the consensus of medical laboratory scientists.

Although studies by Liu and Long [1], by Prellberg and Kramer [2], and by Mondal et al. [9] used the same database (C-NMC dataset), they used different image size and processing, different performance criteria, and did not provide confusion matrices. Therefore, prediction performance comparisons with these earlier studies are not possible. Liu and Long [1] reported a weighted $F_{1}$-score of 0.84 , weighted precision of 0.84 , and weighted recall of 0.85 for their preliminary test set. Prellberg and Kramer [2] reported an average weighted $F_{1}$-score of 0.8789 , an average weighted precision of 0.8791 , and an average weighted recall of 0.9201 for 24 runs of their model in their preliminary test set. Additionally, Prellberg and Kramer [2] concluded that all related works have reported good results, but comparisons are not possible because the datasets are rarely publicly available. Mondal et al. [9] reported that their proposed weighted ensemble model, using the kappa values of the ensemble candidates as their weights, has outputted a weighted $\mathrm{F}_{1}$-score of 0.886 and a balanced accuracy of 0.862 in their preliminary test set. Among the studies that have used publicly available datasets for ALL, comparisons are not possible because the procedures for evaluating classification accuracy differed among studies. Furthermore, all related studies reported so far have used small datasets. Use of a large dataset is essential for an accurate assessment of state-of-the-art classification technology; we hope the C-NMC dataset can meet this need.

The amount of data in the preliminary test set (1,867 records) is much smaller than the amount of data in the training set $(10,661$ records), and the image labeling of the 
preliminary test set data has problems, resulting in lower average accuracy and $\eta$ values the preliminary test set than those of the training set (Table 5). The problem with the image labeling of the preliminary test set data has been explained in the discussion paragraph. Additionally, the $\mathrm{F}_{1}$-score of the preliminary test set in the previous studies $[1,2,9]$ is $0.84-0.886$ and the $F_{1}$-score of the preliminary test set in this study is 0.8894 (obtained by Resnet101-9 ensemble model), indicating that the image labeling of the preliminary test set data needs further confirmation by medical laboratory scientists.

\section{Conclusions}

This Resnet101-9 ensemble model proposed in this study accurately and efficiently classified microscopic images as ALL. The first contribution of this study is the confirmation that an appropriate combination of algorithm hyperparameters for a pretrained Resnet-101 model can obtain high image classification accuracy. The second contribution of this study is the confirmation that the image classification accuracy of an ensemble model can be enhanced by (1) applying a majority voting strategy and by (2) increasing the number of models (e.g., up to nine) integrated in the ensemble model. Additionally, this study investigated the number of image misclassifications made by the Resnet101-9 ensemble model used to classify ALL in microscopic images. When the Resnet101-9 ensemble model was used to classify ALL in a preliminary test set of microscopic images, accuracy was $85.11 \%$, which was superior to the accuracies obtained by the nine trained Resnet-101 individual models (i.e., Resnet-101-8249(\#1), Resnet-101-8184(\#2), Resnet-101-8452(\#3), Resnet-101-8125(\#4), Resnet-101-8061(\#5), Resnet-101-8281(\#6), Resnet-101-8307(\#7), Resnet-101-8002(\#8), and Resnet-101-8216(\#9) models, accuracy ranging from $80.02 \%$ to $84.52 \%)$. Other performance measures obtained for the Resnet101-9 ensemble model (i.e., $86.33 \%$ precision, $91.71 \%$ recall, $72.69 \%$ specificity, and $88.94 \% \mathrm{~F}_{1}$-score) were also superior to those obtained by the nine trained Resnet-101 individual models. That is, the Resnet101-9 ensemble model had superior capability in classifying ALL in microscopic images compared to the nine trained Resnet-101 individual models.

\section{Methods}

The research procedure was collecting data and processing microscopic images for classifying ALL that could be used for model training, selecting the pre-trained Resnet-101 model for transfer learning, using Taguchi method to design the combinations of algorithm hyperparameters for the pre-trained Resnet-101 model, fine-tuning and further training the pre-trained Resnet-101 model to classify ALL in microscopic images, comparing and recording classification performance among different trained Resnet-101 models, inferring the best factor-level combination of algorithm hyperparameters, analyzing algorithm hyperparameters in the trained Resnet-101 model for classifying ALL in microscopic images, generating and selecting multiple trained Resnet-101 models for use in a Resnet101 ensemble model, and, finally, comparing the classification performance of the Resnet101 ensemble model with that of trained Resnet-101 individual models. The detailed steps were as follows. 
Collecting data and processing microscopic images for classifying ALL

The microscopic images in the C-NMC dataset were divided into a training set, a preliminary test set, and a final test set. The training set had 10,661 microscopic images, including 7,272 images of ALL (cancer) cells and 3,389 images of Normal cells. The preliminary test set had 1,867 microscopic images, including 1,219 images of ALL cells and 648 images of Normal cells. Since the ground truth for the final test set was not released, the final test set was not used in the study. Image preprocessing by the dataset authors limited each microscopic image to a single cell and a $450 \times 450$ pixels resolution $[8,10]$.

To maintain compatibility with the CNN-based architecture and the developed software, each microscopic image was processed as a $224 \times 224 \times 3$ image, where 3 is the number of color channels.

\section{Selecting the pre-trained Resnet-101 model for transfer learning}

The most important characteristics of pre-trained CNN models are network accuracy, speed, and size. The choice of a pre-trained network generally involves a tradeoff among these characteristics. Accuracy in classifying images contained in the ImageNet database [11] is the most common measure of the accuracy of networks trained on the database used in the ImageNet large-scale visual recognition challenge (ILSVRC) [12]. Networks that achieve high accuracy on ImageNet are also expected to achieve high accuracy in other natural image datasets that are used to evaluate performance in transfer learning or feature extraction. The Resnet [13] achieved a 3.57\% Top-5 error rate and was the winner of ILSVRC 2015. Therefore, this study selected Resnet-101 (101 layers) for evaluating performance in classifying ALL in microscopic images. Since the Resnet-101 has been trained on more than 1 million images from the ImageNet database used in the ILSVRC, Resnet-101 has learned rich feature representations for a wide range of images and can classify images into 1000 object categories. The image input size for the Resnet-101 is $224 \times 224 \times 3$.

Transfer learning is a machine learning approach in which a model developed for a task is reused as the starting point for a model developed for another task. In transfer learning, a pre-trained CNN model is used to construct a predictive model. Thus, the first step is selecting a pre-trained $\mathrm{CNN}$ model from available models. The second step is reusing the pre-trained $\mathrm{CNN}$ model, and the third and final step is tuning the pre-trained CNN model for a new task. Depending on the input-output pair data available for the new task, the researcher may consider further modification or refinement of the pre-trained CNN model. Transfer learning is typically much faster in a pre-trained $\mathrm{CNN}$ model compared to a $\mathrm{CNN}$ model without pre-training.

\section{Using Taguchi method to design algorithm hyperparameter combinations for the pre-trained Resnet-101 model}

The Taguchi method [14-17] is a statistical experimental method of implementing and evaluating improvements in processes and products. The main principle of the method is to enhance quality by minimizing the cause of variations rather than by eliminating them. The Taguchi method minimizes the number of experiments needed to study a 
large number of design variables. An efficient way to study the effects of several control factors simultaneously is to arrange matrix experiments in orthogonal arrays (OAs). The better factor-level combinations are determined by OAs and signal-to-noise ratios (SNRs).

For the pre-trained Resnet-101 model to achieve high accuracy in classifying ALL in microscopic images, selecting appropriate algorithm hyperparameters was essential. The algorithm hyperparameters for the pre-trained Resnet-101 model in this study were Optimizer, MiniBatchSize, MaxEpochs, and InitialLearnRate. To account for nonlinear effects and to minimize the required number of experiments, a three-level $L_{9}\left(3^{4}\right) \mathrm{OA}$ was used. Therefore, the combinations of algorithm hyperparameters obtained by the three-level $L_{9}\left(3^{4}\right)$ OA were used in a pre-trained Resnet-101 model for classifying ALL in microscopic images.

\section{Fine-tuning and training the pre-trained Resnet-101 model to classify ALL in microscopic images}

To fine-tune a pre-trained Resnet-101 model, transfer learning is often faster and easier than constructing and training a new Resnet-101 model for a new task. Although a pre-trained Resnet-101 model has already learned a rich set of image features, it can be fine-tuned to learn features specific to a new dataset. In this study, the pre-trained Resnet-101 model was fine-tuned to learn features specific to the C-NMC dataset. Since a pre-trained Resnet-101 model can learn to extract a different feature set, the final Resnet-101 model is often more accurate. The starting point for fine tuning deeper layers of a pre-trained Resnet-101 model used for transfer learning is to train the networks with a new C-NMC dataset. Figure 3 is a flowchart of the transfer learning procedure used in the Resnet-101 model.

\section{Comparing and recording classification performance among different trained Resnet-101 models}

The results recorded for the training set and the preliminary test set included (1) accuracy in each run of the experiment, (2) average accuracy in three independent runs, (3) standard deviation in accuracy in three independent runs and (4) $\eta$ value.

Accuracy was defined as the proportion of true positive and true negative results for a population. The concept of SNR was first applied in communications and then in engineering. For engineering applications, a larger SNR $(\eta)$ is preferable and indicates better performance. Taguchi recommended multiplying the common logarithm of SNR by 10 , which obtains the SNR in decibels $(\mathrm{dB})$. In this study, the equation for the

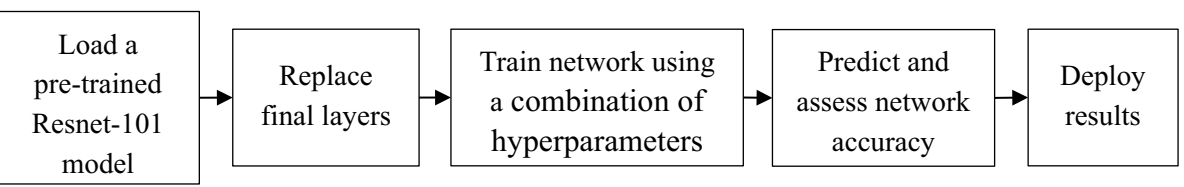

Fig. 3 Flowchart of transfer learning procedure used in the Resnet-101 model 
smaller-the-better characteristic was $\eta=-10 \log (\bar{y}-m)^{2}$, where $\bar{y}=\frac{1}{n} \sum_{t=1}^{n} y_{t}$ (a set of data $y_{1}, y_{2}, \ldots, y_{n}$, accuracy of model training and prediction in each experiment) and $m=1$ (i.e., the accuracy of the target is $100 \%$ ).

\section{Inferring the best factor-level combination of algorithm hyperparameters}

A response table was built to find the best factor-level combination of algorithm hyperparameters by using the $L_{9}\left(3^{4}\right) \mathrm{OA}$ and $\eta$ values. To build the response table, the effects of different factors were set as follows: $E_{f l}=$ average of sum of $\eta_{i}$ for factor $f$ at level $l$, where $f$ is the factor name, $l$ is the level number, and $i$ is the experiment number. After the nine experiments for $L_{9}\left(3^{4}\right)$ were performed, the response table was used to investigate the $\eta$ of each factor level. The response table showed the average $\eta$ of each factor level and maximum average $\eta$ of each factor. The main objective was to use the response table to find the best level for each factor. The best level was defined as the level with the highest $E_{f l}$ value in the experimental region. That is, the best factor-level combination of algorithm hyperparameters was inferred according to the results of the nine experiments, even though not all factor-level combinations (i.e., $3^{4}$ experiments) of algorithm hyperparameters were considered.

\section{Analyzing the algorithm hyperparameters in the trained Resnet-101 model for classifying}

\section{ALL in microscopic images}

The Taguchi experimental design process uses analysis of variance (ANOVA) to identify important control factors by performing the smallest number of experiments. The ANOVA analyses were performed to find the algorithm hyperparameters in the trained Resnet-101 model that significantly affected the most important characteristic, i.e., accuracy in classifying ALL in microscopic images.

\section{Generating and selecting multiple trained Resnet-101 models for integration} in a Resnet101 ensemble model for classifying ALL in microscopic images

The best factor-level combination of algorithm hyperparameters obtained by the response table for the trained Resnet-101 model was used to classify ALL in microscopic images. The trained Resnet-101 models that had the better performance in classifying ALL in microscopic images were then integrated in a Resnet101 ensemble model used to classify ALL in microscopic images in the preliminary testing data set.

\section{Comparing the classification performance of the Resnet101 ensemble model with that of trained Resnet-101 individual models}

Classification performance was compared in the Resnet101 ensemble model and the trained Resnet-101 individual models. Classification performance was compared in terms of accuracy, precision, recall (i.e., sensitivity), specificity, and $\mathrm{F}_{1}$-score values. The five measures were introduced below.

Accuracy is the proportion of true results (both true positive and true negative) in the population. When an information retrieval task is performed, precision is a measure of the relevance of results. Precision is calculated as the positive predictive value (number of true positives over number of true positives plus number of false positives). Another measure 
of information retrieval performance is recall (sensitivity), which is calculated as true positive rate (number of true positives over the number of true positives plus the number of false negatives). Specificity is calculated as true negative rate (number of true negatives over the number of false positives plus the number of true negatives). The $F_{1}$-score is a function of precision and recall and was used to measure prediction accuracy when classes were very imbalanced. The formula used to calculate $\mathrm{F}_{1}$-score in this study was $2 \times$ (precision $\times$ recall $) /($ precision + recall $)[18,19]$.

\section{Abbreviations}

ALL: Acute lymphoblastic leukemia; CNN: Convolutional neural network; LLSVRC: ImageNet large-scale visual recognition challenge; OA: Orthogonal array; SNR: Signal-to-noise ratio; ANOVA: Analysis of variance; SD: Standard deviation.

\section{Acknowledgements}

Not applicable.

\section{About this supplement}

This article has been published as part of BMC Bioinformatics Volume 22 Supplement 5 2021: Proceedings of the International Conference on Biomedical Engineering Innovation (ICBEI) 2019-2020. The full contents of the supplement are available at https://bmcbioinformatics.biomedcentral.com/articles/supplements/volume-22-supplement-5.

\section{Authors' contributions}

YMC, FIC, and JTT contributed equally to algorithm design, programming, and theoretical analysis. YMC, FIC, WHH, and JTT contributed equally to quality control and review of documentation and data. All authors read and approved the final manuscript.

\section{Funding}

Publication costs are funded by the Ministry of Science and Technology, Taiwan, R.O.C., under Grant MOST 109-2221-E-153-005-MY3. This work was supported in part by the Ministry of Science and Technology, Taiwan, R.O.C. under Grant Numbers MOST 109-2221-E-153-005-MY3, MOST 109-2221-E-037-005, and MOST 109-2321-B-037-001. This work was also supported in part by the Headquarters of University Advancement and Intelligent Manufacturing Research Center (iMRC) (National Cheng Kung University) sponsored by the Ministry of Education, Taiwan, R.O.C. The funding bodies played no role in any of the following: study design, data collection, analysis, and interpretation; manuscript writing.

\section{Availability of data and materials}

All data obtained and analyzed in this study are included in this article. Microscopic images of acute lymphoblastic leukemia were obtained from the C-NMC dataset available online at https://doi.org/10.7937/tcia.2019.dc64i46r

\section{Declarations}

Ethics approval and consent to participate

Not applicable.

\section{Consent for publication}

Not applicable.

\section{Competing interests}

The authors declare that they have no competing interests.

\section{Author details}

${ }^{1}$ School of Nursing, Kaohsiung Medical University, Kaohsiung 807, Taiwan. ${ }^{2}$ Superintendent Office, Kaohsiung Medical University Hospital, Kaohsiung 807, Taiwan. ${ }^{3}$ Department of Electrical Engineering, National Kaohsiung University of Science and Technology, Kaohsiung 807, Taiwan. ${ }^{4}$ Department of Healthcare Administration and Medical Informatics, Kaohsiung Medical University, Kaohsiung 807, Taiwan. ${ }^{5}$ Department of Medical Research, Kaohsiung Medical University Hospital, Kaohsiung 807, Taiwan. 'Department of Computer Science, National Pingtung University, Pingtung 900, Taiwan.

Received: 12 December 2021 Accepted: 3 January 2022

Published online: 11 January 2022

\section{References}

1. Liu Y, Long F. Acute lymphoblastic leukemia cells image analysis with deep bagging ensemble learning. ISBI 2019 C-NMC challenge: classification in cancer cell imaging. 2019. p. 113-121.

2. Prellberg J, Kramer O. Acute lymphoblastic leukemia classification from microscopic images using convolutional neural networks. ISBI 2019 C-NMC challenge: classification in cancer cell imaging. 2019. p. 53-61. 
3. Vogado LHS, Veras RDMS, Andrade AR, Araujo FHDD, Silva RRVE, Aires KRT. 2017. Diagnosing Leukemia in blood smear images using an ensemble of classifiers and pre-trained convolutional neural networks. In: 30th SIBGRAPI conference on graphics, patterns and images (SIBGRAPI), Niteroi. 2017. p. 367-373.

4. Rehman A, Abbas N, Saba T, Rahman SIU, Mehmood Z, Kolivand H. Classification of acute lymphoblastic leukemia using deep learning. Microsc Res Tech. 2018;81:1310-7.

5. Shafique S, Tehsin S. Acute lymphoblastic leukemia detection and classification of its subtypes using pre-trained deep convolutional neural networks. Technol Cancer Res Treat. 2018;17:1-7.

6. Kassani SH, Kassani PH, Wesolowski MJ, Schneider KA, Deters R. A hybrid deep learning architecture for leukemic b-lymphoblast classification. In: International conference on information and communication technology convergence, South Korea. 2019. p. 271-276.

7. Loey M, Naman M, Zayed H. Deep transfer learning in diagnosing leukemia in blood cells. Computers. 2020;9(2):1-12.

8. C-NMC-2019 dataset. ALL challenge dataset of ISBI 2019. 2019. https://doi.org/10.7937/tcia.2019.dc64i46r. Accessed 6 Jan 2019.

9. Mondal C, Hasan MK, Jawad MT, Dutta A, Islam MR, Awal MA, Ahmad M, Alyami SA, Ali Moni M.Acute lymphoblastic leukemia detection from microscopic images using weighted ensemble of convolutional neural networks. 2021. p. 1-31. https://doi.org/10.20944/preprints202105.0429.v1.

10. Gupta A, Duggal R, Gehlot S, Gupta R, Mangal A, Kumar L, Thakkar N, Satpathy D. GCTI-SN: geometry-inspired chemical and tissue invariant stain normalization of microscopic medical images. Med Image Anal. 2020;65:101788.

11. Deng J, Dong W, Socher R, Li LJ, Li K, Li FF. ImageNet: a large-scale hierarchical image database. In: Proceedings of the IEEE conference on computer vision and pattern recognition, Florida, USA. 2009. p. 248-255.

12. Russakovsky O, Deng J, Su H, Krause J, Satheesh S, Ma S, Huang Z, Karpathy A, Khosla A, Bernstein M, Berg AC, Li FF. ImageNet large scale visual recognition challenge. Int J Comput Vis. 2015;115:211-52.

13. He K, Zhang X, Ren S, Sun J. Deep residual learning for image recognition. In: Proceedings of the IEEE conference on computer vision and pattern recognition. 2016. p. 770-778.

14. Taguchi G, Chowdhury S, Taguchi S. Robust engineering. New York: McGraw-Hill; 2000.

15. Wu Y. Taguchi methods for robust design. New York: The American Society of Mechanical Engineers; 2000

16. Lee HH. Taguchi methods: principles and practices of quality design. Taiwan: Gau-Lih; 2011.

17. Su CT. Quality engineering. New York: CRC Press; 2013.

18. Chen YM, Chen YJ, Ho WH, Tsai JT. Classifying chest CT images as COVID-19 positive/negative using a convolutional neural network ensemble model and uniform experimental design method. BMC Bioinform. 2021a;22:147.

19. Chen YM, Huang WT, Ho WH, Tsai JT. Classification of age-related macular degeneration and diabetic macular edema using convolutional-neural-network-based transfer learning. BMC Bioinform. 2021 b;22:99.

\section{Publisher's Note}

Springer Nature remains neutral with regard to jurisdictional claims in published maps and institutional affiliations.

- fast, convenient online submission

- thorough peer review by experienced researchers in your field

- rapid publication on acceptance

- support for research data, including large and complex data types

- gold Open Access which fosters wider collaboration and increased citations

- maximum visibility for your research: over $100 \mathrm{M}$ website views per year

At BMC, research is always in progress.

Learn more biomedcentral.com/submissions 